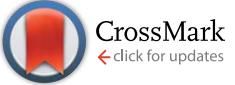

Cite this: RSC Adv., 2016, 6, 48222

\title{
Targeted design of water-based humic substances- silsesquioxane soft materials for nature-inspired remedial applications $\dagger$
}

\author{
Alexander B. Volikov, ${ }^{a}$ Sergei A. Ponomarenko, ${ }^{\text {ab }}$ Alexander Gutsche, ${ }^{c}$ \\ Hermann Nirschl, ${ }^{c}$ Kirk Hatfield ${ }^{d}$ and Irina V. Perminova*a
}

\begin{abstract}
Water-based humic substances-silsesquioxane (HS-SQ) soft materials are synthesized by hydrolysis of (3aminopropyl-triethoxy)-silane in the HS solution. The aggregation dynamics of this system was studied using in situ small-angle X-ray scattering (SAXS) technique, which revealed three consecutive stages in the evolution of the HS-SQ system based on its fractal dimension (D): HS-SQ oligomeric polyelectrolyte complexes with $D<2.5$; loosely bound HS-SQ networks with $2.5<D<3.0$, and densely cross-linked networks with $D>3$ (surface fractals). It was suggested that the reaction time needed for the HS-SQ system to transit from mass to surface fractal stage can be used to control its self-assembly onto a solid support. The corresponding studies have confirmed that the HS-SQ networks could be successfully immobilized onto sand columns only at the aggregation stage with fractal dimensions of $2.5<D_{\mathrm{m}}<3$. This enabled the targeted design of HS-SQ systems capable of guided self-assembly onto the solid support. The corresponding lab column studies have demonstrated successful passive installation of a humic permeable reactive barrier on sand which was capable of intercepting azo dyes from contaminated water. The prospects of using HS-SQ soft materials in nature-inspired remedial technologies and soil restoration are discussed.
\end{abstract}

Received 4th April 2016

Accepted 10th May 2016

DOI: 10.1039/c6ra08636e

www.rsc.org/advances example, when dispersed in oil-contaminated water, humicmodified fine particles can self-assemble into colloidosomes, known as oil-suspended matter aggregates (OSA): this process governs the natural remediation of oil contaminated sediments in coastal environments., ${ }^{4,5}$ Hybrid porous materials, represented by soil aggregates, play a crucial role in both protecting organic carbon and water retention capacity of soils. ${ }^{6}$ Hence, acquiring control over the self-assembly of humic polyelectrolytes, spanning in sizes from 400 to $100000 \mathrm{Da}$, onto the mineral surfaces could present opportunities to develop nature-inspired solutions for environmental remediation and sustainable agriculture.

In this context, incorporation of silanol functionalities into humic molecules looks particularly promising due to the high affinity of silanols to mineral surfaces. Recently, we have demonstrated that silanol-modified humic materials can be sorbed in large quantities (up to $200 \mathrm{mg} \mathrm{g}^{-1}$ ) onto hydroxylated solid supports with well developed surfaces like silica gel and clay. ${ }^{7,8}$ These derived materials demonstrated significantly elevated sorption capacities for organic contaminants (e.g., diazodyes) comparable to that of activated carbon. ${ }^{8}$ Similar hybrid sorbents with immobilized humic layers were described by other authors as well.9-12 Still, these lab-prepared solid sorbents do not meet the challenge of achieving in situ aquifer remediation with injectable reactive barriers (i.e., without excavating contaminated soils or sediments). ${ }^{13,14}$ The same is 
true for restoring the humus content in soils which implies the in situ immobilization of large quantities of organic matter onto soil minerals. To meet these demands, polymer-like soft materials should be developed capable of self assembly into crosslinked networks upon the contact with solid supports (including those with limited surface development, such as sand). We hypothesized that water-based humic substancessiloxane networks could be used for these purposes.

To prove this hypothesis, we have studied the dynamics of cross-linking and aggregation of 3-aminopropyltriethoxysilane (APTES) in the HS solution under varying reaction conditions using the in situ small angle X-ray scattering (SAXS) technique. It provided the unique possibility to monitor the progressive cross-linking of the HS-APTES system over time from loosely linked aggregates with mass fractal dimension towards more densely packed aggregates with surface fractal dimension as it is schematically shown in Fig. 1.

Given that APTES is composed of triethoxy group attached to aminopropyl-radical, the products of its polymerization fall under definition of silsesquioxanes (SQ). The latter refers to all structures with the empirical formulas $\mathrm{RSi}_{3 / 2}$ where $\mathrm{R}$ is hydrogen or any alkyl, alkylene, aryl, arylene, or organofunctional derivatives of alkyl, alkylene, aryl, or arylene groups. ${ }^{15,16}$ We have demonstrated that the HS-SQ network possessed maximum functionality with respect to immobilization onto solid support prior to its transition from mass to surface fractal state. This provided theoretical backgrounds for application of the developed HS-SQ soft materials capable of passive in situ immobilization on solid support (e.g. sand). The sorption performance of the immobilized HS-SQ networks was demonstrated with respect to model contaminant - azo dye. This indicated for the promising perspectives for the in situ applications of HS-SQ networks both for installing "soft matter" reactive barriers in the contaminated aquifers and for increasing the content of humified matter in the organicsdepleted soils.

\section{Experimental}

\subsection{Humic materials}

Coal humic acid (CHP) was prepared by desalination of the potassium humate from leonardite (Powhumus; Humintech Ltd., Germany). It was characterized by the following elemental composition (\% mass on ash-free basis): $\mathrm{C}-53.4, \mathrm{H}$ - 5.15, N-1.31, S - 2.36, O - 37.8; and content of carboxylic groups $\left(\mathrm{mmol} \mathrm{g}^{-1}\right)$ : 4.1. For preparation of HS solutions, a weight of CHP was ionized by few drops of $3 \mathrm{M} \mathrm{NaOH}$, diluted with distilled water, and then adjusted to a desired $\mathrm{pH}$ using 1 $\mathrm{M} \mathrm{HCl}$. All reagents used for this purpose were of analytical grade. Concentration of CHP in experiments solutions varied from 1 to $10 \mathrm{~g} \mathrm{~L}^{-1}$.

\subsection{Humic substances-silsesquioxane networks synthesis}

3-Aminopropyltriethoxy-silane was purchased from Carl Roth $\mathrm{GmbH}$, Germany. It was used to create different APTES : CHP ratios in solution which varied from $1: 4$ to $2: 1$ by weight. APTES was added dropwise to the experimental solution of CHP under continued stirring. $\mathrm{pH}$ of the CHP-APTES solution was adjusted using $1 \mathrm{M} \mathrm{HCl}$, and the total volume was made up to $10 \mathrm{~mL}$ using distilled water. The reaction flask was then hermetically sealed and fixed onto overhead shaker. The experimental solution was then aged and periodically sampled for kinetic measurements using in situ SAXS technique.

\subsection{In situ SAXS and transmission electron microscopy (TEM)}

The SAXS experiments were conducted by means of a modified Kratky camera at the Institute of Mechanical Process Engineering and Mechanics (MVM) of Karlsruhe Institute of Technology (KIT). The camera was equipped with a copper anode (Xray tube $\mathrm{KFL} \mathrm{Cu}$, line focus $0.4 \times 12 \mathrm{~mm}$, Siemens, X-ray generator Kristalloflex 760 Bruker AXS). A photosensitive imaging plate was used as detector. The samples $(0.3 \mathrm{~mL})$ were placed in a quartz capillary and irradiated for three minutes. A detailed description of the camera and the data evaluation are described previously. ${ }^{17}$

TEM images were acquired using a Philips CM 12 microscope operating at $120 \mathrm{kV}$. For these measurements carboncoated grids were briefly dipped into the solution and dried under air.

\subsection{Analysis of scattering data}

The SAXS method is based on the elastic scattering of X-rays at electrons. In a homogeneous medium, the intensity of the scattered X-rays $(I)$ is accordance a function of the scattering vector $(q)$. For a system of $N$ identical particles, the scattering occurs in with the Guinier law:

$$
I(q)=N\left(\Delta \rho^{2}\right) V^{2} \exp \left(-\frac{q^{2} R_{\mathrm{g}}{ }^{2}}{3}\right)
$$

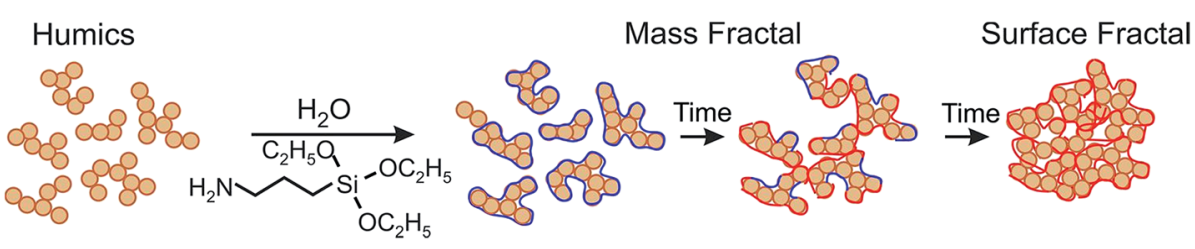

Fig. 1 Schematic interactions between HS and 3-aminopropyltriethoxysilane leading to the formation of aggregates described with fractal dimensions of mass and surface fractals. 
where $R_{\mathrm{g}}$ is the gyration radius of the particles of volume $V$, and $\Delta \rho$ is the difference in electronic density between the particles and the dispersion medium. The Guinier law is valid as long as $R_{\mathrm{g}}$ is small enough (for the range $0<q<1 / R_{\mathrm{g}}$ ). From eqn (3), the radius of gyration may be found from the slope of a plot of $I(q)$ versus $q^{2}$.

For larger $q$, the scattering intensity typically decreases according to a power law. In the power law regime, the concept of fractal geometry can be used to determine structural information on fractals. ${ }^{\mathbf{1 8}, 19}$ Scattering in this regime depends on the geometric structure of the particles in the system. Fresh gels frequently exhibit mass fractal structures. The SAXS intensity from a mass fractal structure shows a power law dependence on $q$ :

$$
I(q) \sim q^{-D_{\mathrm{m}}}
$$

The fractal dimension $1<D_{\mathrm{m}} \leq 3$ can be obtained from the slope of a $\log I v s . \log q$ plot. In case of surface fractals which exhibit a compact interior and a fractal surface of lower density, the power law shows an exponent $3<6-D_{\mathrm{s}} \leq 4$, i.e.

$$
I(q) \sim q^{-\left(6-D_{\mathrm{s}}\right)}
$$

Once the exponent is known, the surface fractal dimension, $D_{\mathrm{s}}$, can be readily calculated.

\subsection{Immobilization of the HS-SQ networks on solid support}

The glass columns prepacked with $100 \mathrm{~mL}$ of quartz sand were used for dynamic sorption. Prior to use, the column was washed with $2 \mathrm{~L}$ of distilled water (MilliQ). A solution of potassium humate CHP was prepared at the concentration of 5 $\mathrm{g} \mathrm{L}^{-1}$, and $20 \mathrm{~mL}$ were transferred into a beaker. A needed aliquot of APTES $(50,100$, or $200 \mu \mathrm{L})$ was added to the beaker under continued stirring to obtain HS-SQ networks with different CHP : APTES ratios: $1: 0.5,1: 1$ and $1: 2(\mathrm{w}: \mathrm{w})$ which were designated as CHP-APTES-50, CHP-APTES-100, and CHP-APTES-200, respectively. Then $\mathrm{pH}$ value was adjusted to 8 using $1 \mathrm{M} \mathrm{HCl}$ and the mixture was aged for $120 \mathrm{~min}$ in case of CHP-APTES-50, for 40 minutes in case of CHP + APTES-100, or immediately introduced into the column in case of CHPAPTES-200.

Each of the aged CHP : APTES mixtures in a volume of $20 \mathrm{~mL}$ was quickly introduced into a sand column at an elution rate of $10 \mathrm{~mL}$ per minute, then $10 \mathrm{~mL}$ of distilled water was added at the same rate to place the mixture in the center of the column. After that the column was hooked up to a flask with distilled water, the flow rate was set to $5 \mathrm{~mL}$ per hour, and flushed overnight. Then the column was washed with distilled water at 1 $\mathrm{mL}$ per minute to remove the residues of non-reacted mixture. Concentration of not-sorbed HS was determined spectrophotometrically at the absorption wavelength of $254 \mathrm{~nm}$. The content of immobilized HS was calculated as a difference between introduced and recovered amount of HS. To remove the immobilized HS-SQ network, $0.01 \mathrm{M} \mathrm{NaOH}(300 \mathrm{~mL})$ was used to flush the column, which desorbed the HS-SQ network from the sand.

\subsection{Dynamic sorption experiments with azo dye Direct Red 81}

Direct Red 81 diazo dye (molecular formula $\mathrm{C}_{29} \mathrm{H}_{19} \mathrm{~N}_{5} \mathrm{Na}_{2} \mathrm{O}_{8} \mathrm{~S}_{2}$, CAS Registry number: 2610-11-9/12237-71-7) was used as a model contaminant. The dye solution in distilled water at a concentration of $25 \mathrm{mg} \mathrm{L^{-1 }}$ was passed through the sand column with the immobilized HS-SQ materials prepared as described above at an elution rate of $1.3 \mathrm{~mL} \mathrm{~min}^{-1}$ until the column was exhausted. The HS-SQ networks with CHP : APTES ratios of $2: 1,1: 1$, and $1: 2$ were immobilized on sand. The void volume of the column was $48 \mathrm{~mL}$. The breakthrough curves of the azo dye were detected by measuring optical density of the solution exiting the column at a wavelength of $511 \mathrm{~nm}$.

\section{Results and discussion}

\subsection{Synthesis of water-based humic substances- silsesquioxane networks}

Upon designing synthesis of water-based HS-SQ networks, we used the unique property of 3-aminopropyl-trialkoxysilanes (APTS) to produce rather stable solutions in water due to formation of cyclic intermediates as it is shown below: $:^{20,21}$

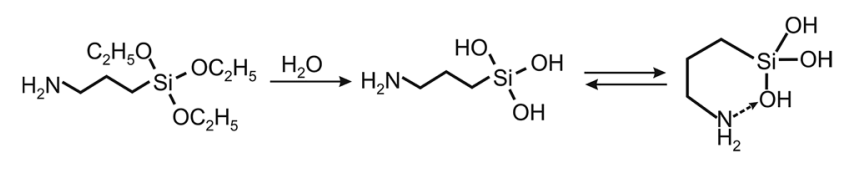

This is unlike other functional organosilanes, which polymerize rather quickly in water with formation of insoluble precipitates of polysiloxanes. Hence, to initiate polymerization of APTS in water, it was necessary to prevent formation of these cyclic intermediates. To achieve that we used negatively charged humic polyanions whose carboxylic functionalities were to outcompete silanols in binding with amine groups as it is shown in the following reaction for 3-aminopropyltriethoxysilan (APTES) used in our studies:

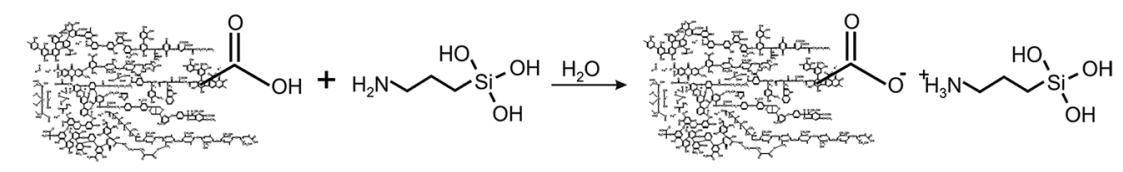


In this case free silanol groups were to start interacting with each other by forming intra- and inter-particle silsesquioxane structures $\left(\mathrm{SiO}_{3 / 2}\right)_{n}$. We have conducted the corresponding reactions at different $\mathrm{pHs}$, reagent ratios, concentration of $\mathrm{HS}$, and in the presence of calcium ions. The motivation was to reveal the primary factors controlling the rate of humic-siloxane network self-assembly and their immobilization properties.

\subsection{In situ SAXS studies of the aggregation dynamics of the aqueous HS-SQ systems}

The SAXS data were acquired over 48 hours after APTES was added to solution of CHP sample. A typical set of SAXS curves is shown in Fig. 2A, the corresponding plot of the power law exponent $n$ versus reaction time is presented in Fig. $2 \mathrm{~B}$, and TEM-images of the CHP-APTES system at 0, 1, 4 and 22 hours reaction time are shown in Fig. $2 \mathrm{C}$.

The SAXS curve of initial solution of CHP did not show any characteristic peaks as indicated by the linearity in log-log plot (Fig. 2A). Its fractal dimensions varied between 2.2 and 2.4, which is indicative that HS in the solution exist as mass fractals producing loose soft networks without a hard core. The resultant $D_{\mathrm{m}}$ values were consistent with the reported findings. ${ }^{22,23}$ The minimum observed $D_{\mathrm{m}}$ values were at an alkaline $\mathrm{pH}$ (2.2 at $\mathrm{pH}$ 9), whereas a decrease in $\mathrm{pH}$ led to an increase in $D_{\mathrm{m}}$ up to 2.4 at $\mathrm{pH}$ 4. This could be explained if HS aggregation were enhanced under acidic $\mathrm{pH}$ conditions due to smaller overall charge and pronounced intramolecular hydrogen bonding. Similar effects were reported in earlier works. ${ }^{24,25}$ The presence of $\mathrm{Ca}^{2+}$ ions in the system caused an increase in $D_{\mathrm{m}}$ value for CHP up to 2.5 , which was to expect, because HS readily react with calcium ions which promotes their aggregation. ${ }^{26}$ (The results are shown in the ESI $\dagger$ ).
The addition of APTES to CHP solution caused a steep increase in a value of fractal dimension (Fig. 2B) which is indicative of substantial aggregation processes occurring in the system. It means that in the course of the CHP-APTES interaction larger aggregates are formed. This process leads to formation of denser core and more porous periphery which is reflected as an increase in fractal dimension. In the low- $q$ regime, a local minimum was observed. Fitting of the corresponding data by means of eqn (3) did not yield reasonable estimates of the gyration radius of the scattering particles. This may indicate that the formed particles were larger than 40-50 $\mathrm{nm}$, which is the upper structural limit of the SAXS camera used in our studies. Hence, the observed inflection might be referred to inter-aggregate scattering of X-rays ${ }^{27}$ resulting from high concentration of humic-siloxane particles. This explanation is in line with the TEM data (Fig. 2C) which show that already at the beginning of the reaction the particles were on the order of 50-70 $\mathrm{nm}$. Then, in the course of APTES condensation, the dense polysilsesquioxane particles were formed which coalesced into larger particle aggregates with sizes 150$250 \mathrm{~nm}$.

As it can be deduced from Fig. 2, in the course of the first hour of the reaction, mostly formation of APTES-HS polyelectrolyte complexes occurs with the fractal dimension close to that of the initial CHP solution. Then, they undergo speedy aggregation followed by an increase in mass fractal dimension from 2.3 up to 3.0. These mass fractals transition further into the surface fractals. The transition of mass to surface fractals can be explained by the fact that the aminoorganosilane bound to humic molecules continued to polymerize in water producing polysilsesquioxane bonds resulting in formation of much denser particles as compared to initial "fluffy" aggregates. These dense particles continued to interact with each other
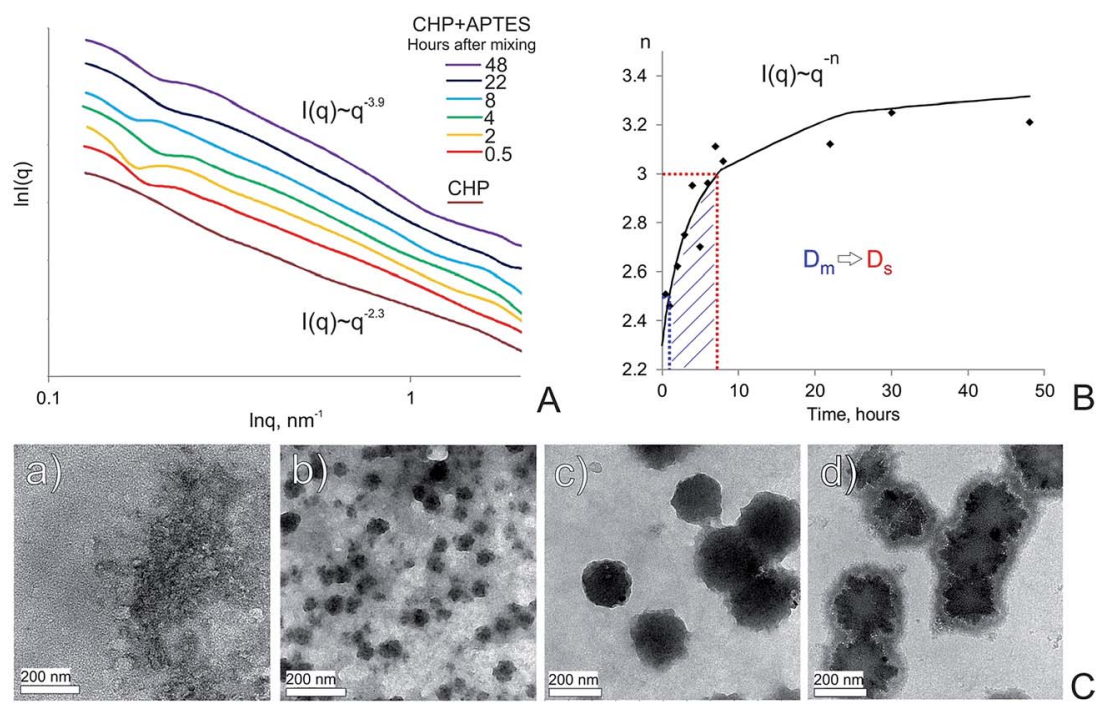

Fig. 2 Evolution of the CHP-APTES system (1: 1 ratio (w : w), $5 \mathrm{~g} \mathrm{~L}^{-1} \mathrm{CHP}, \mathrm{pH}$ 8) over reaction time. (A) SAXS curves at different reaction times (hours) coded with colors: (red - 0.5, yellow -2 , green -4 , cyan -8 , violet -22 , purple -48 ), initial CHP solution - brown; (B) dependence of the slope of log-log plots shown in Fig. 2a on time. Shaded area denotes mass fractal range, transition time of mass to surface fractals ( $D_{\mathrm{m}}$ to $D_{\mathrm{s}}$ respectively) is shown with a red bar; (C) TEM images of CHP-APTES system after 0, 1, 4, and 22 hours of reaction time: (a), (b), (c), and (d), respectively. 
producing aggregates of particles with much smoother surface as compared to initial particles. This process is then reflected as a decrease of the surface fractal dimension of these aggregates (from $D_{\mathrm{s}}=2.7$ down to $D_{\mathrm{s}}=2.1$ ). The obtained X-ray scattering data and TEM imaging allowed us to propose conceptual model of the HS-SQ network formation which is shown in Fig. 3.

\subsection{SAXS studies on dynamics of the CHP : APTES system under different reaction conditions}

To acquire further experimental evidence to the proposed aggregation mechanism, we have probed the dynamics of the CHP : APTES system in time under different reaction conditions by changing $\mathrm{pH}$, reagent ratios, concentration of $\mathrm{HS}$ and $\mathrm{Ca}$ ions. Overview of the results of in situ SAXS responses to the above parameters are summarized in Fig. 4 and detailed data are summarized in Tables S1-S4 in the ESI. $\dagger$

It can be seen that the HS-SQ system behaved in a similar manner over a broad $\mathrm{pH}$ range in a sense that the mass fractals were formed at the initial interaction stage, which were further transformed into the surface fractals (Fig. 4A). The observed transformation did not imply a phase transition, but it was accompanied by a substantial change in the material properties, such as porosity, surface area, and activity, which decreased along with a transition of mass to surface fractals. Hence, the time needed for such a transition can serve as a characteristic parameter of the system to control or even exploit its functional properties. From this point of view it is of importance to note that the maximum transition time from mass to surface fractals ( 30 hours) was characteristic to the CHP-APTES system with initial $\mathrm{pH} 6$, which corroborates well the silicon chemistry: the minimum hydrolysis and siloxane bond formation rate at $\mathrm{pH} 6 .^{28-30}$

A decrease in this transition time (which is equivalent to the higher hydrolysis and siloxane formation rate) was observed both along with acidification of the system ( 2 hours at $\mathrm{pH} 4)$ and even more with the alkanization of the system (immediately at $\mathrm{pH}$ 9). This behavior can be explained by acidic and basic catalysis of the hydrolytic processes which undergo organoalkoxysilanes in aqueous solutions which is schematically shown below: ${ }^{30}$

$$
\begin{aligned}
& \geqslant \mathrm{Si}_{1}-\mathrm{OH}+\mathrm{HO}-\mathrm{Si}_{2} \underset{\mathrm{H}_{2} \mathrm{O}}{\stackrel{\mathrm{HO}^{-}}{\rightleftarrows}}>\mathrm{Si}_{1}-\mathrm{O}-\mathrm{Si}_{2}=+\mathrm{H}_{2} \mathrm{O} \\
& >\mathrm{Si}_{1}-\mathrm{OH}+\mathrm{HO}-\mathrm{Si}_{2} \subsetneq \underset{\mathrm{H}_{2} \mathrm{O}}{\stackrel{\mathrm{H}^{+}}{\rightleftarrows}}>\mathrm{Si}_{1}-\mathrm{O}-\mathrm{Si}_{2} \subsetneq+\mathrm{H}_{2} \mathrm{O}
\end{aligned}
$$

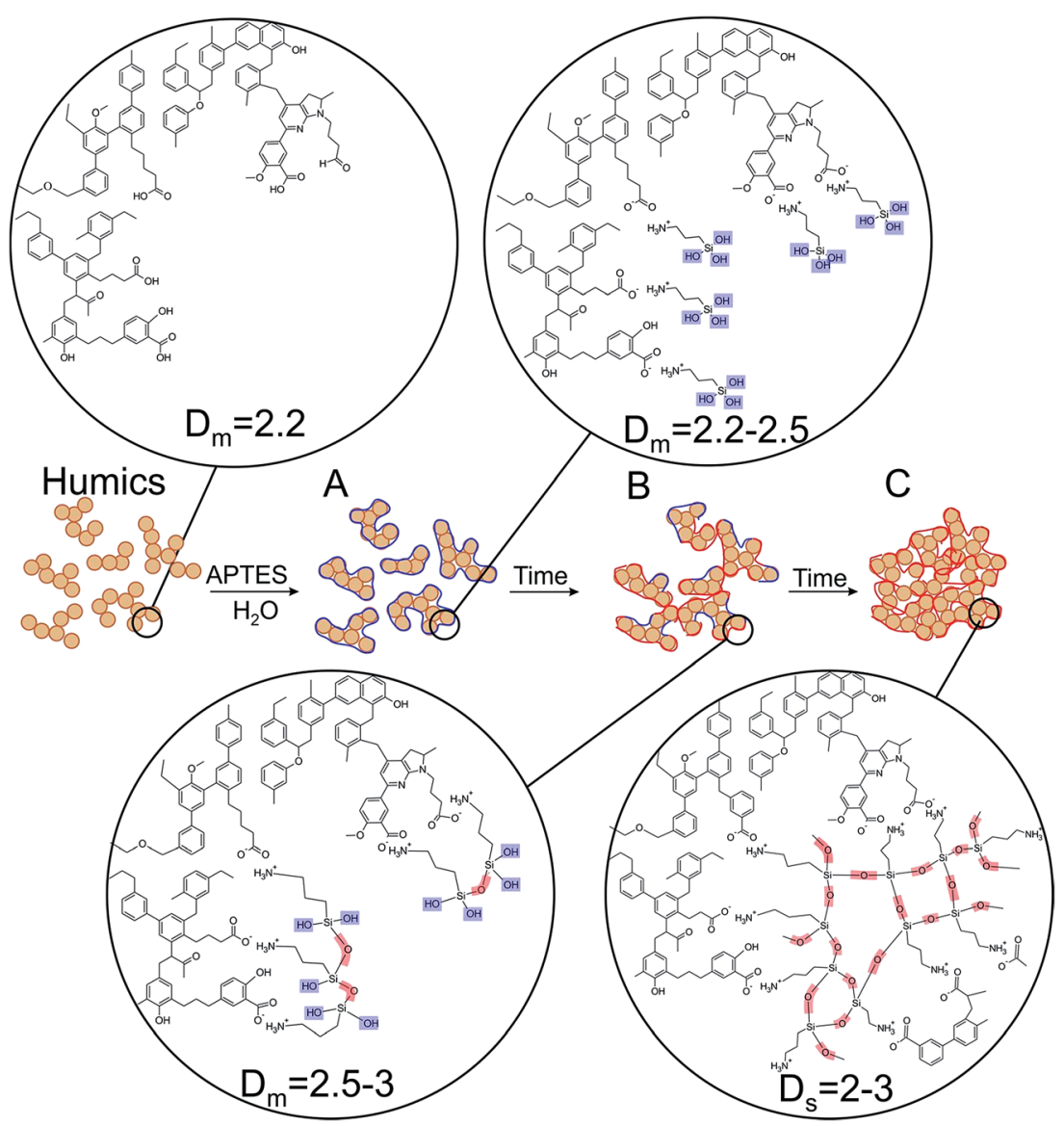

Fig. 3 Conceptual model of the water-based HS-SQ network formation and evolution in time: the first insert shows a fragment of HS network with $D_{m}$ of 2.2, (A) shows formation of CHP-APTES complexes rich in silanol groups (marked in blue) with low $D_{m}$ values, (B) shows initial siloxane bonding (marked in red) resulting in aggregate formation with high $D_{\mathrm{m}}$ values, (C) shows branched silsesquioxane cross-linking $\left(D_{\mathrm{m}}\right.$ to $D_{\mathrm{s}}$ transition). 

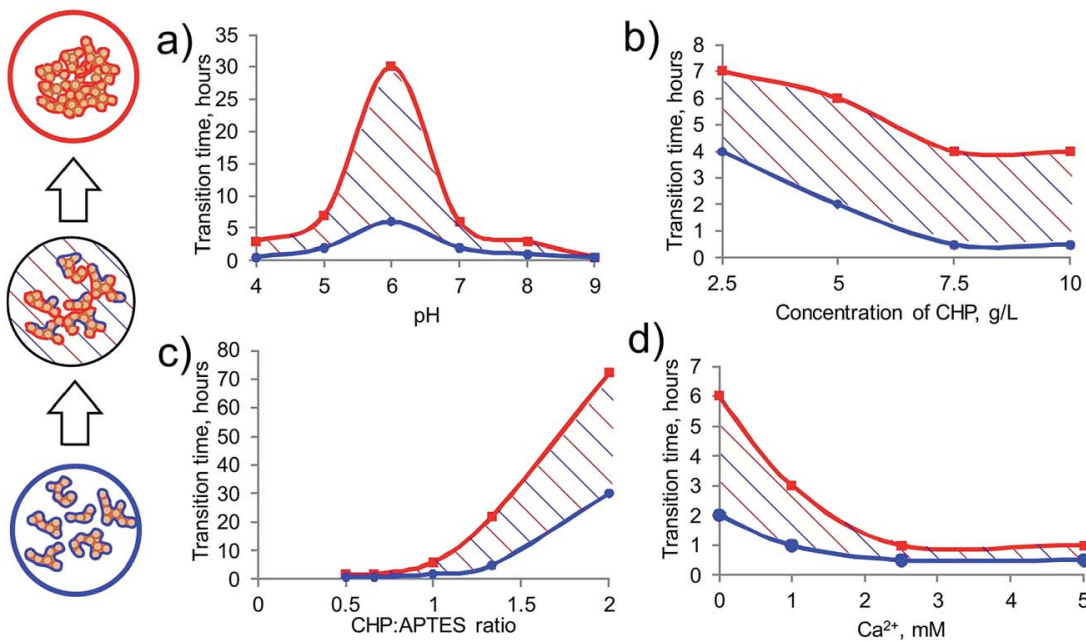

d)

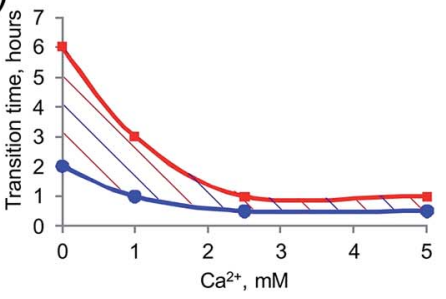

Fig. 4 The dependence of the transition time of the mass to surface fractals conversion in the CHP-APTES system (highlighted in red), and of the time needed to reach the mass fractal dimension $D_{m}=2.5$ (highlighted in blue) on different reaction parameters: (a) $p H$ (CHP : APTES $=1: 1, C H P$ concentration $\left.=5 \mathrm{~g} \mathrm{~L}^{-1},\left[\mathrm{Ca}^{2+}\right]=0\right)$; (b) concentration of $\mathrm{CHP}\left(\mathrm{pH}=7, \mathrm{CHP}: \mathrm{APTES}=1: 1,\left[\mathrm{Ca}^{2+}\right]=0\right)$, (c) the ratio of CHP : APTES (pH =7, CHP

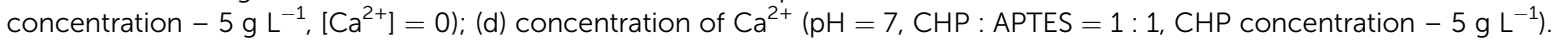

This is a reason that an impact of other parameters on formation of humic-siloxane networks, was examined at $\mathrm{pH} 7$ and varied concentrations of $\mathrm{HS}, \mathrm{Ca}$, and the reagent ratio (CHP : APTES).

Concentration of HS had less pronounced effect on the polymerization rate as compared to a change in $\mathrm{pH}$ value (Fig. 4B). Still, its increase accelerated the transition of the system from mass to surface fractal state. Much more significant was the reagent ratio (CHP : APTES). An increase in APTES proportion significantly accelerated the system aging accompanied by transition from mass to surface fractal particles: at the CHP : APTES ratio of $1: 2$, it occurred in only 2 hours, whereas at the $2: 1$ ratio, the transition took place after 72 hours. The further increase in CHP proportion in the system ( $4: 1$ ratio) slowed down the transition time so dramatically that it was not reached even after a month of aging time: the fractal dimension did not exceed the $D_{\mathrm{m}}$ value of 2.5. The solution was stable over this month and there was no precipitate observed. An addition of $\mathrm{Ca}$ ions has substantially accelerated formation of aggregates (Fig. 4D). This corroborates the expected behavior of the system while it is known that calcium promotes aggregation of both HS and APTES in aqueous solutions due to formation of insoluble salts. ${ }^{26}$ As a result, the HS-SQ network was least stable in the presence of calcium.

The relationships found corroborate the conceptual model of the evolution of HS-SQ network proposed in Fig. 3. Their examination suggests that the HS-SQ system under study possesses maximum functionality with regard to its immobilization capacities when it reaches the state of poorly cross-linked large aggregates rich in silanol groups (stage $\mathrm{C}$ ). This allowed us to define the optimum operational conditions shown with shaded areas in Fig. 4. Within these areas the poorly crosslinked voluminous aggregates are formed rich in silanol groups capable of further cross-linking into 3D polysilsesquioxane networks. This makes those systems the best candidates for immobilizing onto solid supports depleted with surface binding sites, e.g., sand. The key for preparing the CHPAPTES systems with those properties is to use appropriate reaction time (in the range of blue and red lines) for the given reaction conditions.

\subsection{Immobilization of the HS-SQ networks onto the solid supports}

To demonstrate how the aging time of the CHP : APTES mixture before its contact with the solid support impacts the immobilizing performance, we prepared CHP : APTES mixtures at $\mathrm{pH} 8$ and injected them into the column at three different stages of HS-SQ networks formation: (1) immediately after mixing at the stage of interpolyelectrolyte complexes; (2) after aging time which did not exceed the transition time of mass to surface fractals; and (3) after aging time exceeding transition time of mass to surface fractals. We used high elution rates to inject the CHP : APTES mixtures into the middle of the column, then the flow rate was slowed down to approach those of the ground water, and the system was washed continuously with distilled water. The intent was to simulate active injection of the preconditioned HS-SQ network via a borehole followed by its passive migration and immobilization onto the solid support of the aquifer. The immobilization performance of the CHPAPTES system used at different aging times is illustrated in Fig. 5.

These experiments allowed us to determine aging times that provide for best HS-SQ network immobilization at different CHP : APTES ratio $(2: 1,1: 1$, and $1: 2)$ at the given $\mathrm{pH}$. For the systems under study they accounted for (in minutes) 120 , 40 , and 5, respectively, which corroborates with the mass-tosurface fractal transition time determined for the corresponding systems from the scattering data (Fig. 3).

A capturing performance of the same HS-SQ networks with three different reagent ratios was estimated based on the 


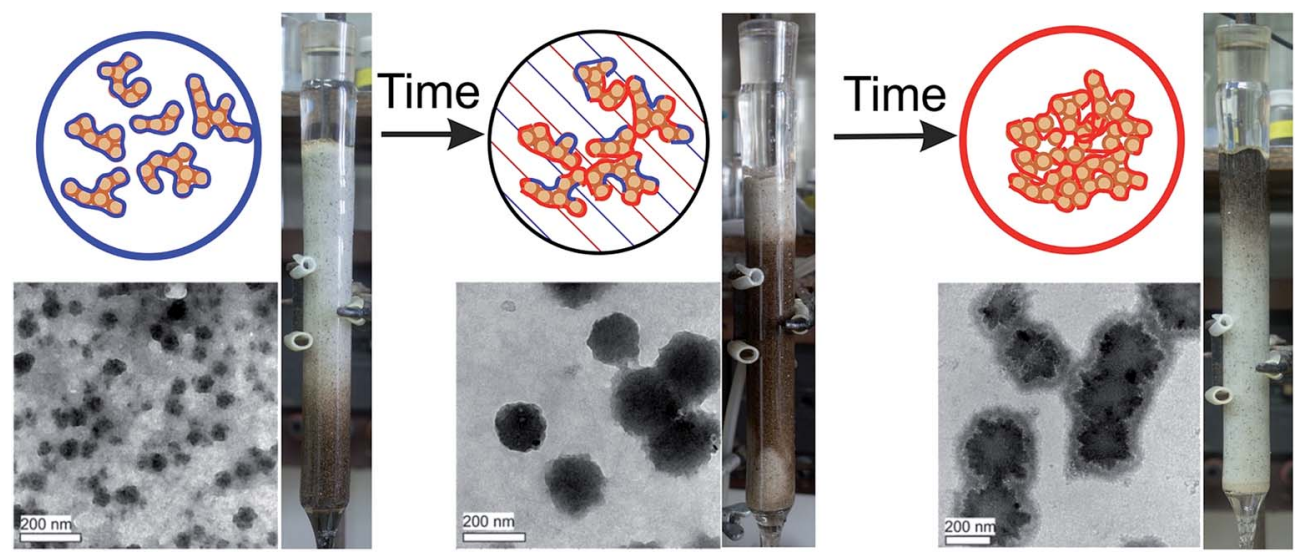

Fig. 5 Immobilization of the HS-SQ networks onto the sand column after different aging times (CHP : APTES $=1: 1$, concentration of $C H P=5 \mathrm{~g}$ $\mathrm{L}^{-1}, \mathrm{pH}$ 8): left panel - injected at aging time 0 (immediately after mixing): no retardation on the column; middle panel - injected after 40 min: the humic layer was immobilized in the middle of the column, right panel - injected after 2 hours: the precipitate was formed and settled in the upper part of the column.

amount of the reagent leached from the column. The corresponding values accounted for (in $\%$ of the introduced mass) 21,81 , and 96 for the CHP : APTES systems with reagent ratios of $2: 1,1: 1$, and $1: 2$, respectively (the data are presented in Table 1). This indicates that an increase in APTES proportion improves performance of the system. Of importance is that the immobilization of the HS-SQ network onto the sand did not impact its hydraulic conductivity. Hence, the developed system has good prospects as a liquid medium for installation of humic permeable reactive barriers (HPRBs) onto granular support of the contaminated aquifers. From technological point of view it was necessary to find a way for removing HPRBs from aquifer support. The corresponding experiments on desorption of HPRB from sand have demonstrated that the HS-SQ system can be completely removed (washed out) from sand by diluted alkali (e.g., $0.01 \mathrm{M} \mathrm{NaOH})$. We have found that a switch of washing solution from distilled water to $0.01 \mathrm{M}$ $\mathrm{NaOH}$ lead to complete desorption of the HS-SQ network from the sand. In practice it means that a diluted alkali can be pumped in into the installation well; and after desorption of the HS-SQ system it can be pumped out of another well located downstream in the same aquifer. Overall, the results obtained demonstrate a facile in situ immobilization and removal of HPRB onto granular aquifer support (e.g. sand) using guided self-assembly of the water-based HS-SQ networks.
To evaluate functional properties of the immobilized HS-SQ networks with respect to contaminant sequestration, we used the azo dye Direct Red 81, which is a dibasic sodium salt. Given that both the selected azodye and humic polyelectrolytes are negatively charged at neutral $\mathrm{pH}$, we expected increased sorption of the diazodye onto HS-SQ networks with the higher content of positively charged APTES. The corresponding column experiments are shown in Fig. 6A. The sorption isotherms are shown for three different CHP : APTES ratios (Fig. 6B). After the column was saturated with the azo dye, we conducted desorption of azodye using distilled water as an eluent. This was done to estimate amount of azo dye which was strongly bound to the HS-SQ polymers. The corresponding sorption-desorption parameters are summarized in the two last columns of Table 1.

From Fig. 6 and Table 1 it can be deduced that the azo dye was poorly sorbed on pure sand, but it was retained in much larger quantities by the immobilized HS-SQ materials; moreover, the amount of sorbed azodye increased along with the amount of APTES in the HS-SQ network and with the total amount of the humic material immobilized onto sand.

This might be indicative of leading electrostatic interactions between the azo dye and the HS-SQ soft material immobilized onto sand: only small amount of dye was sorbed by CHP-APTES50 , which carried overall negative charge (the observed sorption might be referred to hydrophobic binding); whereas much

Table 1 Sorption-desorption characteristics ${ }^{a}$ of the azo dye Direct Red 81 (AD) onto the HS-SQ materials immobilized on sand at different CHP : APTES ratios

\begin{tabular}{lllll}
\hline $\begin{array}{l}\text { Column } \\
\text { treatment }\end{array}$ & $\begin{array}{l}\text { Amount of retained } \\
\text { CHP, mg }\end{array}$ & $\begin{array}{l}\text { Amount of retained } \\
\text { CHP, \% of total }\end{array}$ & $\begin{array}{l}\text { Amount of sorbed } \\
\text { AD, mg }\end{array}$ & $\begin{array}{l}\text { Amount of desorbed } \\
\text { AD, mg }\end{array}$ \\
\hline Pure quartz sand & 0 & 0 & 0.28 & 0.08 \\
CHP : APTES of $1: 2$ & 12 & 21 & 2.14 & 0.23 \\
CHP : APTES of $1: 1$ & 68 & 81 & 14.92 & 0.06 \\
CHP : APTES of $2: 1$ & 92 & 96 & 3.68
\end{tabular}

${ }^{a}$ For sorption experiments the elution rate was $5 \mathrm{~mL} \mathrm{~h}^{-1}$ for desorption $-1 \mathrm{~mL} \mathrm{~h}^{-1}$. Desorption was conducted using distilled water. 
a)

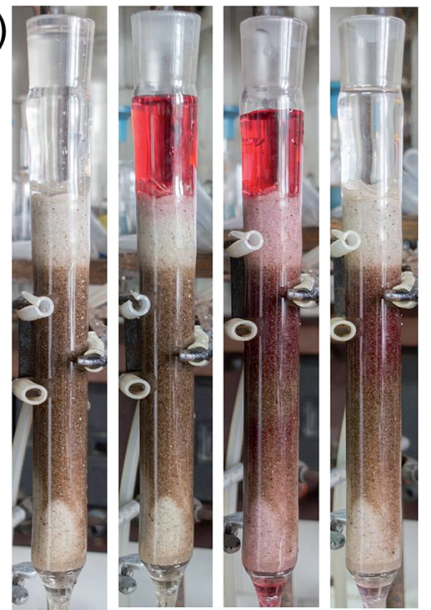

b)

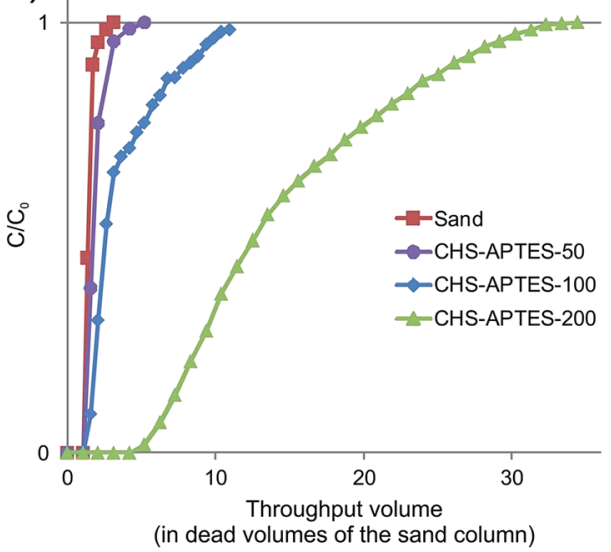

Fig. 6 Dynamic sorption of Direct Red 81 azo dye onto the HS-SQ soft material immobilized in situ onto sand. (a) Experimental design; (b) dynamic isotherms for the CHP : APTES ratios of $2: 1,1: 1$, and $1: 2$, highlighted in violet, blue, and green, respectively. Concentration of azodye is $25 \mathrm{mg} \mathrm{L}^{-1}, \mathrm{pH}$, elution rate is $1.3 \mathrm{~mL} \mathrm{~min}^{-1}$, void column volume is $48 \mathrm{~mL}$.

larger amounts of azodye were retained at the CHP-APTES-200 which was characterized by an excess of amino groups of organosilane and carried overall positive charge. The obtained results are in good agreement with the reported data on enhanced sorption of dyes on humics-modified solid sorbents., ${ }^{9,12}$

It should be specifically stressed that as in case of the removal of the immobilized HS-SQ networks from the granular aquifer support, which we discussed above, the sorbed azodye can be also completely removed from the solid support by washing it off with $0.01 \mathrm{M} \mathrm{NaOH}$. In our column experiments we have found that the azodye elutes together with the HS-SQ materials upon washing with $0.01 \mathrm{M} \mathrm{NaOH}$ (the results are not shown here).

To estimate maximum amount of HS which can be immobilized onto the solid support with a use of the HS-SQ networks under study, we conducted batch sorption experiments with silica gel (the isotherms are shown in Fig. S1 in the ESI $\dagger$ ). The estimated values of sorption capacity were as high as $200 \mathrm{mg}$ of HS per $1 \mathrm{~g}$ of silica gel which corresponds to $9 \%$ of organic carbon. These estimates are similar to those reported for silanol derivatives in our previous publications. ${ }^{7,31}$ Of even more importance is that they are comparable to those for organic rich geosorbents, such as mollisols, sediments, and others. ${ }^{32}$ Hence, the developed water-based HS-SQ networks might be also used as soil meliorants for restoring humus content in the degraded soils.

\section{Conclusions}

The presented results on the synthesis and fractal properties of the water-based HS-SQ networks provide theoretical basis for the targeted design of the HS-SQ soft materials capable of guided self-assembly aimed at in situ immobilization on granular supports, e.g. sand, under conditions of aqueous solution. Detailed studies using in situ SAXS and TEM on aggregation dynamics of these materials during aging allowed us for the first time to define experimental conditions, which enable immobilization of appreciable amounts of humic materials on sand in the form of HS-SQ networks. These conditions converge to a choice of appropriate aging time of the HS-SQ system for achieving a right level of loosely bound cross-linked aggregates, prior it switches to the condensed state of the surface fractals. Strict observation of these conditions is needed to apply the HSSQ materials as potent remedial agents for installation of injectable humic PRBs. The good prospects are shown for targeted design of the HS-SQ materials with tunable remedial properties aimed at enhancing their performance for the specific applications. For example, for removal of positively charged contaminants (such as cations), the HS-SQ materials with predominant contribution of humic component should be used (e.g., CHP-APTES-50), the sorption of anions is more preferred onto siloxane-rich materials (e.g., CHS-APTES-200), whereas the highest sorption of non-ionogenic hydrophobic compounds is expected onto neutral CHP : APTES networks (e.g., CHP-APTES-100). The immobilized HS-SQ networks can passively intercept and accumulate the pertinent contaminants. Upon exhaustion they can be easily removed by flushing with solution of diluted alkali. Green synthesis, high performance, ease of installation and removal from the solid support are among the most attractive features of these new soft matter agents with promising applications for nature-inspired remedial technologies such as passively PRBs, biomimetic catalysis, and guided self-assembly of polymer-like materials. ${ }^{33,34}$ They can be also applied for advanced soil restoration and carbon sequestration technologies implying a use of humics-based systems..$^{35}$

\section{Acknowledgements}

The authors would like to acknowledge Dr Vladimir V. Volkov (Institute of Crystallography of RAS) for very helpful discussions 
on the SAXS results. Alexander Volikov would like to express his gratitude to the program of academic exchanges between Lomonosov Moscow State University and the Karlsruhe Institute of Technologies (KIT) for support of his stay at the Institute of Mechanical Processes of KIT (Germany) where he conducted in situ SAXS experiments. The studies on SAXS data interpretation, synthesis and immobilization of humic-siloxane networks were supported by the Russian Science Foundation (grant \#1614-00167).

\section{References}

1 P. MacCarthy, Soil Sci., 2001, 166, 738-751.

2 I. V. Perminova, K. Hatfield and N. Hertkorn, Use of Humic Substances to Remediate Polluted Environments: From Theory to Practice, Springer Verlag, Dordrecht, the Netherlands, 2005.

3 M. Klučákova, Environ. Eng. Sci., 2014, 31, 612-620.

4 J. Sun and X. A. Zheng, J. Environ. Monit., 2009, 11, 18011809.

5 E. H. Owens, G. A. Sergy, C. G. Guenette, R. C. Prince and K. Lee, Spill Sci. Technol. Bull., 2003, 8, 257-272.

6 J. Six, E. T. Elliott and K. Paustian, Soil Biol. Biochem., 2000, 32, 2099-2103.

7 A. B. Volikov, V. A. Kholodov, N. A. Kulikova, O. I. Philippova, S. A. Ponomarenko, E. V. Lasareva, A. M. Parfyonova, K. Hatfield and I. V. Perminova, Catena, 2016, 137, 229-236.

8 A. B. Volikov, S. A. Ponomarenko, A. I. Konstantinov, K. Hatfield and I. V. Perminova, Chemosphere, 2016, 145, 83-88.

9 A. G. S. Prado, B. S. Miranda and G. V. M. Jacintho, Surf. Sci., 2003, 542, 276-282.

10 A. G. S. Prado, B. S. Miranda and J. A. Dias, Colloids Surf., A, 2004, 242, 137-143.

11 G. Szabo, S. L. Prosser and R. A. Bulman, Chemosphere, 1990, 21, 729-739.

12 L. K. de Oliveira, E. F. Molina, A. L. A. Moura, E. H. de Faria and K. J. Ciuffi, ACS Appl. Mater. Interfaces, 2016, 8, 14781485.

13 R. H. Karol, Chemical Grouting, Marcel Dekker, New York, 2nd edn, 1990.

14 M. J. Truex, E. M. Pierce, M. J. Nimmons and S. V. Mattigod, Evaluation of in situ grouting as a potential remediation method for the Hanford central plateau deep vadose zone. Prepared for the U.S. Department of Energy under Contract DE-AC05-76RL01830, PNNL, Richland, Washington, 2011.
15 R. H. Baney, M. Itoh, A. Sakakibara and T. Suzuki, Chem. Rev., 1995, 95, 1409-1430.

16 R. Sodkhomkhum and V. Ervithayasuporn, Polymer, 2016, 86, 113-119.

17 V. Goertz, A. Gutsche, N. Dingenouts and H. Nirschl, J. Phys. Chem. C, 2012, 116, 26938-26946.

18 K. D. Keefer and D. W. Schaefer, Phys. Rev. Lett., 1986, 56, 2376-2379.

19 P. W. Schmidt, J. Appl. Crystallogr., 1991, 24, 414-435.

20 P. R. Moses, L. M. Wier, J. C. Lennox, H. O. Finklea, J. R. Lenhard and R. W. Murray, Anal. Chem., 1978, 50, 576-585.

21 H. Ishida, S. Naviroj, S. K. Tripathy, J. J. Fitzgerald and J. L. Koenig, J. Polym. Sci., Part B: Polym. Phys., 1982, 20, 701-718.

22 R. L. Wershaw, P. J. Burcar, C. L. Sutula and B. J. Wiginton, Science, 1967, 157, 1429-1431.

23 P. K. Pranzas, R. Willumeit, R. Gehrke, J. Thieme and A. Knöchel, Anal. Bioanal. Chem., 2003, 376, 618-625.

24 N. Senesi, Soil Sci., 1999, 164, 841-856.

25 J. A. Rice and J. S. Lin, Environ. Sci. Technol., 1993, 27, 413414.

26 N. Kloster, M. Brigante, G. Zanini and M. Avena, Colloids Surf., A, 2013, 427, 76-82.

$27 \mathrm{P}$. Schmidt, Use of scattering to determine the fractal dimension. The Fractal Approach to Heterogeneous Chemistry, Wiley Interscience, Chichester, UK, 1989.

28 F. D. Osterholtz and E. R. Pohl, J. Adhes. Sci. Technol., 1992, 6, 127-149.

29 B. Arkles, J. R. Steinmetz, J. Zazyczny and P. Mehta, J. Adhes. Sci. Technol., 1992, 6, 193-206.

30 F. Beari, M. Brand, P. Jenkner, R. Lehnert, H. J. Metternich, J. Monkiewicz and H. W. Siesler, J. Organomet. Chem., 2001, 625, 208-216.

31 I. V. Perminova, L. A. Karpiouk, S. A. Ponomarenko, K. Hatfield, A. I. Konstantinov, N. Hertkorn and A. M. Muzafarov, Colloids Surf., A, 2012, 396, 224-232.

32 S. Endo, P. Grathwohl, S. B. Haderlein and T. C. Schmidt, Environ. Sci. Technol., 2009, 43, 393-400.

33 K. Hagenhoff, J. Dong, B. Chowdhry, L. Torres and S. Leharne, Physics and Chemistry of the Earth, Parts $A / B / C$, 2012, 37-39, 18-25.

34 F. Sannino, R. Spaccini, D. Savy and A. Piccolo, J. Hazard. Mater., 2013, 261, 55-62.

35 A. Piccolo, R. Spaccini, A. Nebbioso and P. Mazzei, Environ. Sci. Technol., 2011, 45, 6697-6702. 